\title{
Composite fermions in periodic and random antidot lattices
}

\author{
J. H. Smet, D. Weiss, ${ }^{*}$ and K. von Klitzing \\ Max-Planck Institut für Festkörperforschung, Heisenbergstraße 1, D-70569 Stuttgart, Germany \\ P. T. Coleridge and Z. W. Wasilewski \\ Institute for Microstructural Sciences, National Research Council of Canada, Ottawa, Ontario, Canada K1A OR6 \\ R. Bergmann and H. Schweizer \\ 4. Physikalisches Institut, Universität Stuttgart, Pfaffenwaldring 57, D-70569 Stuttgart, Germany \\ A. Scherer \\ Department of Physics, California Institute of Technology, Pasadena, California 91125
}

(Received 27 March 1997)

\begin{abstract}
The longitudinal and Hall magnetoresistance of random and periodic arrays of artificial scatterers, imposed on a high-mobility two-dimensional electron gas, were investigated in the vicinity of Landau level filling factor $\nu=1 / 2$. In periodic arrays, commensurability effects between the period of the antidot array and the cyclotron radius of composite fermions are observed. In addition, the Hall resistance shows a deviation from the anticipated linear dependence, reminiscent of quenching around zero magnetic field. Both effects are absent for random antidot lattices. The relative amplitude of the geometric resonances for opposite signs of the effective magnetic field and its dependence on illumination illustrate enhanced soft wall effects for composite fermions. [S0163-1829(97)06231-0]
\end{abstract}

In 1989 , Jain, ${ }^{1}$ guided by the phenomenological similarity between the fractional and integer quantum Hall effect (FQHE and IQHE), introduced the concept of composite fermions (CF's) as an elegant alternative theory to describe the prominent features of the FQHE at filling fractions $\nu=p /(2 p \pm 1)$, where $p$ is an integer. The electron system is subjected to a statistical gauge field transformation which maps it into a new fermionic system of composite particles, each consisting of an electron and two flux quanta and experiencing both the external magnetic field $B$ and nucleated flux. Subsequently, it is hoped for that a mean-field approximation to the transformed Hamiltonian, such that the CF's are subjected to an effective magnetic field $B_{\text {eff }}$ equal to $B-B_{\nu=1 / 2}$, captures the essential physics of the original problem. The series of FQHE liquids around half filling may then be regarded as a manifestation of the IQHE of CF's. Furthermore, the prediction of Halperin et $\mathrm{al}^{2}$ of the existence of a CF Fermi sea with a well-defined Fermi surface at $\nu=1 / 2$ showed the way towards quasiclassical experiments, such as commensurability oscillations in $1 \mathrm{D}$ or $2 \mathrm{D}$ periodically modulated systems and transverse magnetic focusing, ${ }^{3-6}$ to demonstrate the existence of these quasiparticles and their quasiclassical cyclotron motion.

The strength of this class of experiments in providing support for the composite-fermion picture stems from the availability of an easily variable scaling parameter, either the periodicity of periodically modulated systems or the center-tocenter constriction spacing of focusing geometries. Since our understanding of the asymptotic low-energy behavior of the composite-fermion dispersion is on a weak footing, they in addition benefit from the advantage that the matching condition between cyclotron radius and this scaling parameter is independent of the effective mass. Here, square periodic and fully random antidot superlattices have been investigated. In addition to the longitudinal resistivity, previously studied by Kang et $a l .{ }^{7}$ for periodic arrays, the Hall resistivity is also investigated. We find a quenching of the Hall resistivity around $\nu=1 / 2$, ascribed to "channeling" trajectories of composite fermions. These commensurability features disappear in random arrays of antidots, demonstrating that the anomalies around $\nu=1 / 2$ are solely due to the presence of a periodic potential. This and previous experiments ${ }^{7-9}$ make the case that a mean-field approximation to the Hamiltonian with a Chern-Simons gauge field transformation embodies much of the physics of the original electron problem. Furthermore, the influence of changes in the potential landscape due to additional illumination are reported.

The samples were prepared from two high-mobility GaAs- $\mathrm{Al}_{x} \mathrm{Ga}_{1-x} \mathrm{As}$ heterojunctions with the twodimensional electron gas (2DEG) located $160 \mathrm{~nm}$ (sample I) and $200 \mathrm{~nm}$ (sample II), and the $\delta$ doped layer $100 \mathrm{~nm}$ underneath the sample surface. The samples were shaped into Hall bar geometries using standard lithographic techniques and either alloyed $\mathrm{AuGe} / \mathrm{Ni} / \mathrm{Au}$ or In contact pads. Prior to electron-beam lithography and after illumination with a red light-emitting diode (LED), the areal density $n_{e}$ and electron mobility $\mu$ at zero magnetic field and $1.3 \mathrm{~K}$ were $2.2 \times 10^{11} \mathrm{~cm}^{-2}$ and $3.4 \times 10^{6} \mathrm{~cm}^{2} / \mathrm{Vs}$ (sample I), and $1.3 \times 10^{11} \mathrm{~cm}^{-2}$ and $4.3 \times 10^{6} \mathrm{~cm}^{2} / \mathrm{Vs}$ (sample II). Antidot lattices with periodicities $a$ of $400 \mathrm{~nm}$ to $700 \mathrm{~nm}$ and aperiodic arrays (see lower inset of Fig. 2) with equal average antidot densities were imposed by electron-beam lithography and a subsequent, approximately $130 \mathrm{~nm}$ deep, reactive ion etch (RIE) with $\mathrm{SiCl}_{4}$. The cell areas of the Wigner-Seitz cell construction for each of the antidots of a random lattice form a Poisson distribution with a mean $\bar{s}$ equal to $a^{2}$ and 


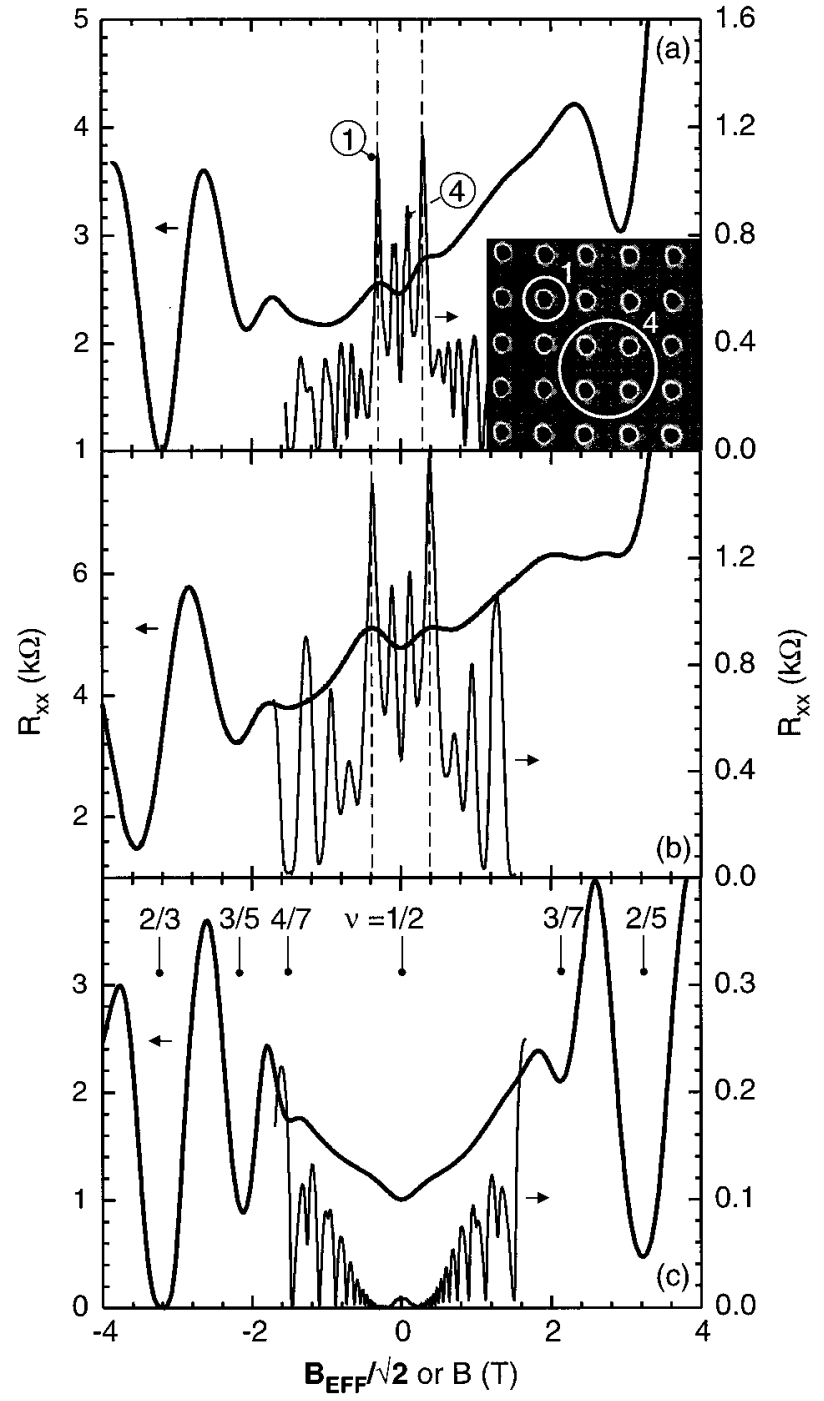

FIG. 1. The magnetoresistance $R_{x x}$ on sample I of antidot superlattices with lattice periods $a$ equal to 500 (a) and $400 \mathrm{~nm}$ (b) near $\nu=1 / 2$ (left axes, thick solid curves) and near zero magnetic field (right axes, thin solid curves) at $450 \mathrm{mK}$. Also shown in (c) is the magnetoresistance near $\nu=1 / 2$ and $B=0$ for an unpatterned reference section of the Hall bar. For ease of comparison, the magnetic field scale of the CF curves has been divided by $\sqrt{2}$ and shifted horizontally to make $B_{\nu=1 / 2}$ coincide with $B=0$ $\left(B_{\text {eff }}=B-B_{\nu=1 / 2}\right)$. The carrier concentrations, obtained after illumination with a red LED, are, respectively, $2.14 \times 10^{11}$ (a), $2.30 \times 10^{11}(\mathrm{~b})$, and $2.19 \times 10^{11} \mathrm{~cm}^{-2}$. The inset shows a scanning electron micrograph of a $400 \mathrm{~nm}$ antidot array.

$\sigma / \bar{s}=0.53$, where $\sigma$ is the standard deviation. Clustering of antidots is not prevented and therefore the local antidot density may deviate considerably from the average value. Using standard ac lock-in techniques with the external magnetic field applied perpendicular to the 2DEG, four-point magnetoresistance measurements were carried out in a $30 \mathrm{~T}$ Bitter magnet with ${ }^{3} \mathrm{He}$-insert or an $18 \mathrm{~T}$ superconducting magnet with dilution refrigerator.

Figure 1 presents the magnetoresistance $R_{x x}$ at a temperature of $450 \mathrm{mK}$ near zero magnetic field and around half filling for antidot superlattices with periods $a$ of 400 and 500 $\mathrm{nm}$ and for a reference section devoid of antidots on one of the Hall bars. Low field maxima in the electron traces (right axes) are anticipated whenever the electron cyclotron radius is commensurate with the lattice, such that a fraction of the carriers follow pinned trajectories that encircle an integer number of antidots and do not contribute to the transport process. ${ }^{3}$ Both lattices exhibit two prominent electron commensurability peaks, labeled 1 and 4 in Fig. 1(a).

Accepting the validity of the composite-fermion-ChernSimons theory, the ground state and low-energy excitations at filling factor $\nu=1 / 2$ can be described by a modified Fermiliquid theory. ${ }^{2}$ For a magnetic field $B$ deviating a small amount $B_{\text {eff }}$ from the field $B_{\nu=1 / 2}$ corresponding to half filling, composite fermions follow quasiclassical circular orbits with a cyclotron radius

$$
R_{c, \mathrm{CF}}=\frac{\hbar k_{F, \mathrm{CF}}}{e B_{\mathrm{eff}}},
$$

where the Fermi wave vector $k_{F, C F}$ is related to the electron density by $k_{F, \mathrm{CF}}=\sqrt{4 \pi n_{e}}$ and differs by a factor $\sqrt{2}$ from the electron Fermi wave vector due to complete spin polarization of the composite fermion sea at these high magnetic fields. The magnetic field value $B_{\nu=1 / 2}$ at half filling was determined from the well-developed fractional quantum Hall minimum at $\nu=2 / 3$. This value was in all cases within less than $100 \mathrm{mT}$ of the local minimum in $R_{x x}$ near $\nu=1 / 2$. The geometric resonance of CF's around one antidot is indeed observed in Fig. 1. Its magnetic field position properly scales with the lattice period and lines up, after taking into account complete spin polarization, with the electron resonances. The density inhomogeneity and the much smaller CF mean free path have been pointed to as the main culprits for the broadening of the geometric resonance features. ${ }^{7,8}$

The periodic arrangement of antidots also causes the Hall resistance $R_{x y}$, shown in Fig. 2 for a periodic and random lattice, to deviate from its expected linear dependence around half filling. Its behavior reminds us of the quenching of the Hall resistance around zero magnetic field in antidot lattices ${ }^{3}$ and in small mesoscopic junctions. ${ }^{10}$ In the former, the Hall field is impaired due to trajectories channeling over many periods along the main axes of the antidot array. Besides a slope change at $\nu=1 / 2$, a shift to higher resistance values is noticeable and has been predicted in Ref. 11. It is noteworthy that the emperical relationship $R_{x x} \propto B d R_{x y} / d B$ (Ref. 12) also reproduces the exact position of the geometric resonances.

Both this and the previously reported antidot experiment ${ }^{7}$ consistently yield an asymmetry around half filling with a reduced strength of the commensurability peak for positive effective magnetic field. It has been attributed to soft wall effects and originates from the CF property that the nonuniform electrostatic potential in the vicinity of soft walls translates into a spatially modulated effective magnetic field. ${ }^{9,11}$ The 2D modulation potential may be influenced by additional illumination of the sample with a red LED. Figure 3 demonstrates the evolution of the commensurabilty peaks for electrons and CF's with successive illumination steps. The asymmetry gradually becomes more pronounced. For a true antidot potential, illumination merely steepens the soft walls and according to the quasiclassical theory for the motion of CF's outlined in Ref. 11 one should recover the symmetry of 


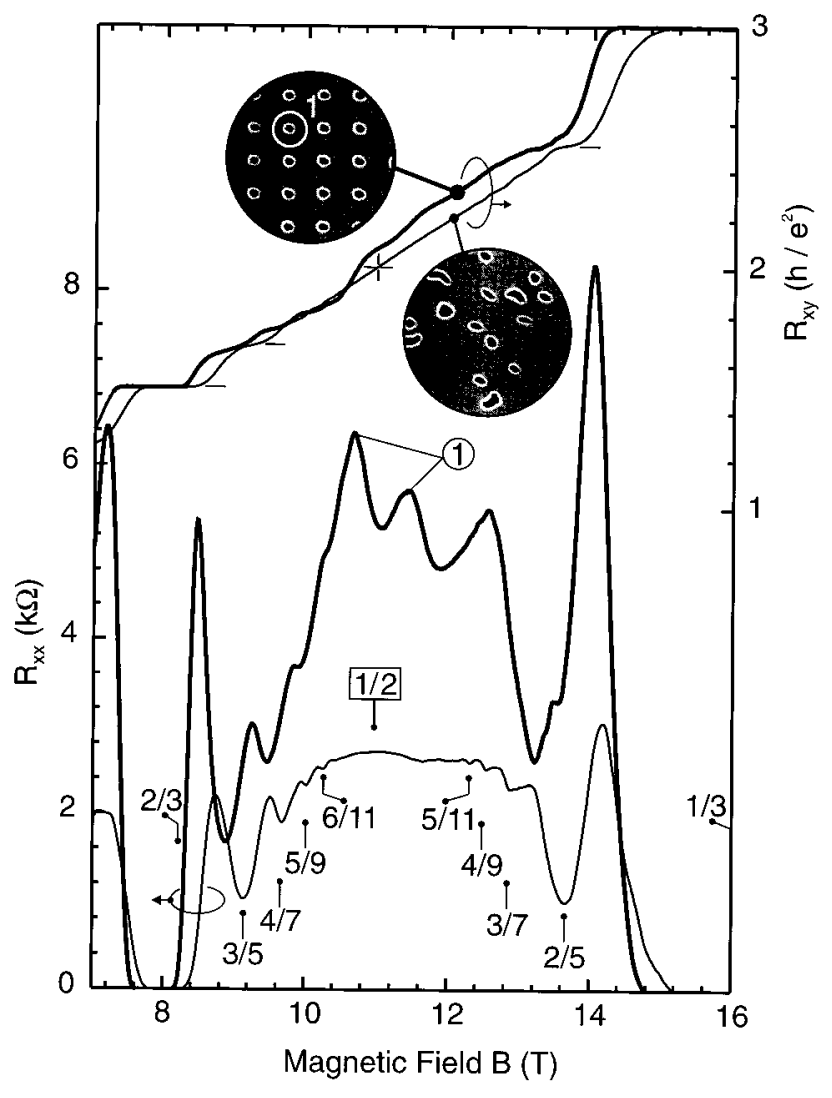

FIG. 2. The Hall and longitudinal resistance on sample II of a $400 \mathrm{~nm}$ periodic antidot array (thick lines) and a random lattice (thin lines) of equal average antidot density at $30 \mathrm{mK}$. A shift to higher values and quenching of the Hall resistivity in the periodic lattice is apparent. The cross marks filling factor $\nu=1 / 2$. Insets show scanning electron micrographs of the lattices. The resistance maximum adjacent to the commensurability peaks on the high magnetic field side appears to be the precursor of the resistance peak between filling factors $\nu=2 / 5$ and $\nu=3 / 7$. This interpretation is supported by the illumination dependent behavior shown in Fig. 3(b).

the geometric resonances around zero effective magnetic field. This apparent contradiction between theory and experiment may however be resolved by considering the low field data in Fig. 3(a). Limitations of the RIE-etching process in producing antidots with large depth to diameter aspect ratios prevented us from reaching the $200 \mathrm{~nm}$ deep 2DEG. As a result, we hypothesize that upon sufficient illumination one no longer retains a true antidot potential but, although presumably still relatively strong, 2D modulation not necessarily with any fully depleted zones [see inset of Fig. 3(b)]. The electron mean free path, extracted from the zero field resistance, increases from $0.75 \mu \mathrm{m}$ to more than $12 \mu \mathrm{m}$ upon illumination. For an antidot potential, the electron mean free path ought to remain comparable to the lattice period. Initially the electron commensurabilty features hardly shift on the magnetic field axis [top four curves in Fig. 3(a)], but become weaker and get more and more submerged in Shubnikov-de Haas oscillations. The two lower traces no longer display the characteristic antidot peaks, but show structure reminiscent of the commensurability oscillations observed in weakly modulated 2DEG's. ${ }^{5,6}$ The minima of the
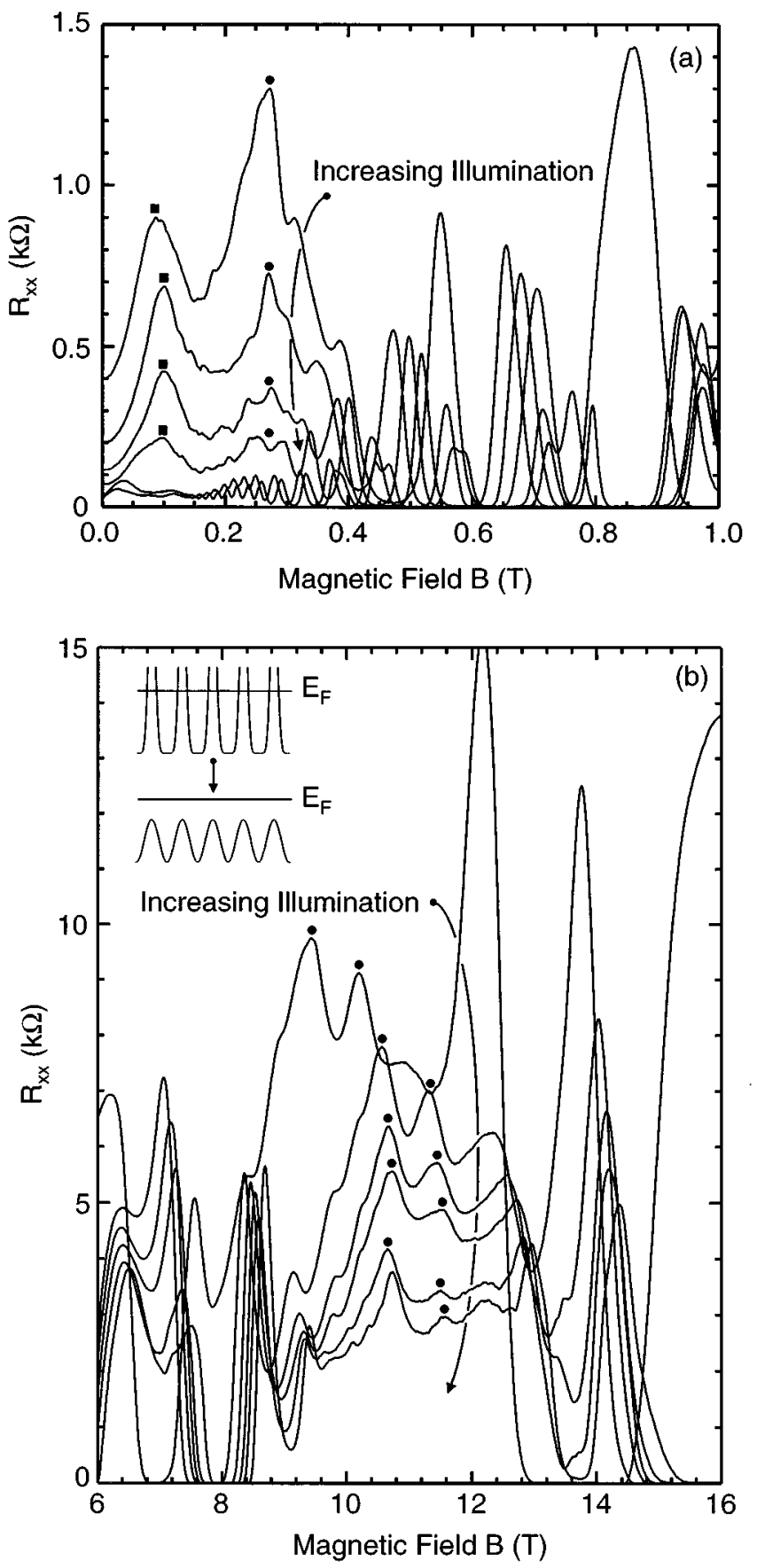

FIG. 3. The dependence of the longitudinal magnetoresistance for the $400 \mathrm{~nm}$ periodic antidot array of Fig. 2 on the amount of illumination with a red LED at low (a) and high (b) magnetic fields. Commensurability features encircling 1 and 4 antidots are marked with solid circles and rectangles, respectively. The inset shows schematically the expected evolution of the potential profile from the low magnetic field data in (a).

oscillations are close to the expected values given by $2 R_{c}=a(\lambda-1 / 4)$ with $\lambda$ an integer. We project that when tuning the potential landscape from a true antidot potential towards weak $2 \mathrm{D}$ modulation the asymmetry may increase rather than decrease.

In summary, commensurability oscillations of composite fermions in antidot lattices have been observed, confirming previous results. ${ }^{7}$ In addition, the Hall resistance exhibits 
quenching at $\nu=1 / 2$. While they are absent for random arrays, both effects can be ascribed to the periodic arrangement of the antidots. The experiments, in particular the asymmetric antidot peaks around $\nu=1 / 2$ as well as the deviations in the Hall resistance, emphasize the role of soft wall potentials, as has been previously pointed out in recent theoretical work. $^{11}$

We are indebted to R. Fleischmann for enlightening discussions, P. Zawadski for initial sample characterization, and
H. Gräbeldinger, M. Riek, B. Schönherr, and U. Waizmann for help with sample preparation. This work has been supported by the Bundesministerium für Bildung und Forschung, the European Community project "Human Capital and Mobility, Physics in High Magnetic Fields,' the European Canadian Mesoscopics Initiative, and a NATO Collaborative Research Grant. The transport measurements at high magnetic fields were carried out in part at the National High Magnetic Field Laboratory sponsored by the National Science Foundation and the state of Florida.
*Also with Institut für Experimentelle und Angewandte Physik, Universität Regensburg, D-93040 Regensburg, Germany.

${ }^{1}$ J. K. Jain, Phys. Rev. Lett. 63, 199 (1989).

${ }^{2}$ B. I. Halperin, P. A. Lee, and N. Read, Phys. Rev. B 47, 7312 (1993).

${ }^{3}$ D. Weiss, M. L. Roukes, A. Menschig, P. Grambow, K. von Klitzing, and G. Weimann, Phys. Rev. Lett. 66, 2790 (1991).

${ }^{4}$ H. van Houten, C. W. J. Beenakker, J. G. Williamson, M. E. I. Broekaart, P. H. M. van Loosdrecht, B. J. van Wees, J. E. Mooij, C. T. Foxon, and J. J. Harris, Phys. Rev. B 39, 8556 (1989).

${ }^{5}$ D. Weiss, K. von Klitzing, K. Ploog, and G. Weimann, Europhys. Lett. 8, 179 (1989); R. R. Gerhardts, D. Weiss, and K. von Klitzing, Phys. Rev. Lett. 62, 1173 (1989).

${ }^{6}$ R. W. Winkler, J. P. Kotthaus, and K. Ploog, Phys. Rev. Lett. 62, 1177 (1989).
${ }^{7}$ W. Kang, H. L. Störmer, L. N. Pfeiffer, K. W. Baldwin, and K. W. West, Phys. Rev. Lett. 71, 3850 (1993).

${ }^{8}$ R. L. Willett, R. R. Ruel, K. W. West, and L. N. Pfeiffer, Phys. Rev. Lett. 71, 3846 (1993).

${ }^{9}$ J. H. Smet, D. Weiss, R. H. Blick, G. Lütjering, K. von Klitzing, R. Fleischmann, R. Ketzmerick, T. Geisel, and G. Weimann, Phys. Rev. Lett. 77, 2272 (1996).

${ }^{10}$ M. L. Roukes, A. Scherer, S. J. Allen, Jr., H. G. Craighead, R. M. Ruthen, E. D. Beebe, and J. P. Harbison, Phys. Rev. Lett. 59, 3011 (1987).

${ }^{11}$ R. Fleischmann, T. Geisel, C. Holzknecht, and R. Ketzmerick, Europhys. Lett. 36, 167 (1996).

${ }^{12}$ S. H. Simon and B. I. Halperin, Phys. Rev. Lett. 73, 3278 (1994), and references therein. 